\title{
Métodos de evaluación de competencias en serious games: estudio y análisis sobre su estado actual \\ Skill assessment methods in serious games: a state of the art study and analysis
}

\author{
Juan Antonio Caballero-Hernández ${ }^{1}$, Manuel Palomo-Duarte², Juan Manuel Dodero ${ }^{3}$ \\ juanantonio.caballero@uca.es,manuel.palomo@uca.es, juanma.dodero@uca.es \\ ${ }^{1}$ Grupo de investigación EVALfor \\ Universidad de Cádiz \\ ${ }^{2,3}$ Departamento de Ingeniería Informática \\ Puerto Real, España \\ Universidad de Cádiz \\ Puerto Real, España
}

\begin{abstract}
Resumen- Los serious games o videojuegos educativos son videojuegos con propósitos de aprendizaje. Los jugadores deben obtener competencias y resultados de aprendizaje en una experiencia de juego. Éstos serán evaluados conforme al comportamiento desarrollado durante la partida. Sin embargo, la evaluación en serious games es un procedimiento más complejo que el de otros instrumentos e-Learning debido a una serie de factores y particularidades, como el contexto del videojuego o su género. Esta publicación hace una recopilación de los métodos de evaluación en serious games utilizados y propuestos en la literatura científica actual. Se presenta una definición para cada uno de estos métodos, así como un análisis general sobre los factores que tienen en cuenta y sus posibles similitudes. Finalmente, se presentan las conclusiones del estudio y las necesidades detectadas sobre las que se podría profundizar en el futuro.
\end{abstract}

Palabras clave: Videojuegos educativos; Aprendizaje basado en juegos; Edutainment; Evaluación de competencias; e-Learning

Abstract- Serious games or educational games are video games with learning purposes. Players must obtain skills and learning outcomes playing a videogame. They are usually assessed according to their behaviors during the gameplay. Unfortunately, assessment in serious games is a more complex process than other e-Learning assessment processes due to specific features, like game context or genre. This paper compiles assessment methods for serious games detected in the current scientific literature. A definition for each found method and an overall analysis about considered features and possible similarities are provided. Finally, conclusions of the study and future research lines are presented.

Keywords: Serious games; Game-Based Learning; Edutainment; Skill assessment; e-Learning

\section{INTRODUCCIÓN}

En los últimos años, el uso de las nuevas tecnologías de la información y la comunicación se ha ido incrementando y consolidando en nuestra vida diaria a un ritmo cada vez mayor. Estos avances también han afectado a los entornos educativos, de forma que se encuentren mejoras en el desarrollo de herramientas de aprendizaje (Graven \& MacKinnon, 2005). Esto ha llevado a que tanto universidades como centros educativos de todo tipo se hayan visto en la necesidad de introducir cambios y avances tecnológicos. Una evidencia del aumento del uso de tecnologías de la información en entornos educativos es la masiva implantación de sistemas de gestión de aprendizaje o "Learning Management Systems” (LMS), como Blackboard o Moodle.

Para los estudiantes universitarios, estas tecnologías facilitan el acercamiento a la información y desarrollo de competencias tanto académicas como profesionales, lo cual proporciona una oportunidad para crear nuevos contextos de aprendizaje, más flexibles y personalizados (Traverso-Ribón et al., 2016). Entre estos nuevos contextos destaca especialmente el uso de los videojuegos, ya que de forma habitual se han asociado en exclusiva a entornos de ocio y entretenimiento. La utilización de los videojuegos brinda nuevas posibilidades en la creación y desarrollo de prácticas que impliquen activamente al alumnado (Berns et al., 2016).

Los videojuegos constituyen una industria que genera millones de euros al año (Van Eck, 2006) y ya supera a otras industrias de entretenimiento como el cine o la televisión. Según su objetivo, se pueden distinguir entre videojuegos de entretenimiento y videojuegos educativos (Djaouti et al., 2011), también conocidos como "serious games” o "Game Based Learning” (GBL). Los videojuegos de entretenimiento buscan únicamente la diversión del jugador, mientras que los serious games también incluyen propósitos de aprendizaje.

Debido a sus propósitos educativos, los serious games pueden considerarse como herramientas e-Learning. Sin embargo, estos juegos tienen algunas características que los hacen diferentes y añaden complejidad respecto al resto de sistemas e-Learning a la hora de ser evaluados. Hay que tener en cuenta un amplio número de factores en la evaluación de serious games, tanto genéricos (objetivos del aprendizaje, contexto en el que se desarrollará el juego, etc.) como específicos (género del juego, evaluación durante o tras el gameplay, etc.) Teniendo en cuenta la mayor complejidad de evaluación que proponen los serious games, se antoja necesaria una recopilación de métodos de evaluación de este tipo de videojuego para conocer el estado actual de la literatura.

Esta publicación busca detectar propuestas de métodos de evaluación en la literatura científica. El resto del trabajo está estructurado de la siguiente manera: la sección 2 presenta un contexto general sobre los serious games y los métodos de evaluación de competencias. La sección 3 describe los 
métodos identificados en la literatura y un ejemplo de aplicación para cada uno. En la sección 4 se hace un análisis y discusión sobre los métodos encontrados. Finalmente, en la sección 5 se listan las conclusiones y se identifican posibles líneas de investigación futuras.

\section{CONTEXTO}

A continuación se presenta una revisión sobre los serious games y diversos conceptos relacionados, así como su relación con los métodos de evaluación de competencias.

\section{A. Serious games y conceptos relacionados}

La terminología usada para referirse a las experiencias de aprendizaje basadas en videojuegos educativos puede ser diferente según el autor, ya que existen una serie de expresiones que en ocasiones se utilizan como sinónimos o bien como conceptos complementarios. Algunos de los términos más usados en este campo son: "serious games", "edutainment”, “Game Based Learning (GBL)”, "Digital Game Based Learning” (DGBL) y "applied games”. Aunque el concepto de e-Learning está ampliamente relacionado, es un término más general ya que hace referencia a experiencias de aprendizaje mejoradas gracias al uso de computadoras, aprendizaje basado completamente en computadoras y tecnología interactiva (Hodson et al., 2001).

Los serious games son introducidos en 1970 por Abt (1970). En su libro, Abt propone juegos y simulaciones para mejorar la educación tanto dentro como fuera del aula. Define los serious games como "juegos con un explícito propósito educativo y no para ser jugados únicamente con motivos de entretenimiento". Posteriormente, esta definición es revisada por Zyda (2005), que añade los conceptos de juegos de computador y herramienta para el aprendizaje en múltiples entornos: "desafío mental jugado por ordenador según unas reglas específicas, y que tiene en cuenta el entretenimiento para promover la formación gubernamental o corporativa, de educación, salud, normas públicas y objetivos de comunicación estratégica”. Hoy en día, la mayoría de experiencias de aprendizaje basadas en serious games siguen la tendencia del uso de videojuegos.

El término "edutainment” se refiere a la educación a través del entretenimiento y fue muy popular durante la década de los 90 con el crecimiento del mercado multimedia (Michael \& Chen, 2006). Para Buckingham y Scanlon (2000), "Edutainment” es un género híbrido que usa estilos más amenos ya que se basa mayormente sobre materiales visuales, narrativa o formatos basado en juegos, por lo que se requiere el uso de una pedagogía interactiva.

El concepto de GBL es idéntico al de serious games para algunos autores como Corti (2006), donde se refiere a GBL como el uso de serious games en procesos de aprendizaje. Indica que GBL trata de tomar ventaja del poder de los juegos de ordenador para involucrar a los usuarios finales en un propósito específico, así como desarrollar nuevos conocimientos y habilidades. Además, posibilita a los estudiantes el llevar a cabo tareas y experimentar situaciones que de otra forma no serían factibles o posibles. Por otro lado, los DGBL están estrechamente relacionados con GBL, ya que hacen referencia al mismo tipo de experiencias pero indican explícitamente el uso de juegos de ordenador (Prensky, 2001).
Finalmente, “applied games” es un término relativamente nuevo en la literatura y que puede considerarse como una evolución de los serious games (Schmidt et al., 2015). Este tipo de juegos son definidos como "la implementación de una materia, motivada y diseñada a lo largo de una transferencia centrada tanto en el contexto como en los usuarios de conceptos de diseño y aspectos del mundo de juego".

Todos estos conceptos se solapan y según los diferentes autores las definiciones pueden desde usarse como sinónimos hasta discrepar. En esta publicación se hace referencia al término “serious games”, englobando en él todo tipo de experiencias de aprendizaje basadas en videojuegos.

\section{B. Evaluación en experiencias de aprendizaje basadas en serious games}

La evaluación de los procesos de aprendizaje en el actual marco de la educación superior se centra sobre la obtención por parte del alumno de competencias y resultados de aprendizaje, no sólo sobre las actividades individuales realizadas (Palés-Argullós et al., 2010). Por tanto, es necesario que muchos sistemas de evaluación establecidos sean redefinidos y adopten nuevas estrategias de evaluación que tengan en cuenta estos aspectos (Ibarra \& Rodríguez, 2011).

Con el aumento de las nuevas tecnologías dentro de los sistemas educativos, la evaluación de competencias adquiridas por parte de alumnos mediante el uso de ordenadores lleva siendo redefinida varios años. Recientes estudios han explorado cómo diferentes tecnologías y herramientas de eLearning pueden mejorar la calidad de las evaluaciones tradicionales añadiendo feedback sobre las competencias desarrolladas por los estudiantes, además de simplificar y automatizar tareas de corrección/evaluación y obtención de informes (Rodríguez et al., 2012; Caballero-Hernández et al., 2014; Balderas et al., 2015).

Los serious games deben dar soporte a la obtención de competencias por parte del jugador, por lo que la evaluación de la actuación realizada es de vital importancia. Tanto los serious games como los videojuegos en general pueden contener (y asiduamente lo hacen) tests de efectividad dentro del propio juego (Bente \& Breuer, 2009). Esta efectividad se mide en base al progreso de los jugadores a través del juego, de forma que acumulen puntos y experiencia que les dote de las habilidades necesarias para enfrentarse a nuevos retos en niveles posteriores. Este enfoque es muy efectivo ya que integra juegos y pedagogía, por lo que permite proporcionar feedback inmediato al jugador e implementar niveles de adaptabilidad para el usuario (Michael \& Chen, 2005; Enfield et al., 2012).

\section{DESCRIPCIÓN DE LOS MÉTODOS DE EVALUACIÓN EN SERIOUS GAMES EN LA LITERATURA}

Ante la gran cantidad de artículos dedicados a los serious games y la falta de información sobre los métodos de evaluación utilizados, en este apartado se listan una serie de métodos de evaluación en serious games propuestos por varios conjuntos de autores diferentes. Los nombres de los métodos no se han traducido para evitar pérdida de precisión en futuras búsquedas o consultas. Además, se aportan referencias de serious games que aplican el método evaluativo correspondiente. 
- In-Process assessment: también conocida como evaluación "In-Game”. Analiza dentro del propio juego cómo, cuándo y por qué un jugador ha tomado una serie de decisiones. Este análisis se realiza dentro del propio juego y durante el mismo (Michael \& Chen, 2005). Por ejemplo, una aventura gráfica en la que se analicen las distintas acciones y decisiones tomadas por el jugador (Moreno-Ger et al., 2007).

- Completion assessment: se centra en la medición del éxito que obtuvo el jugador tras completar el videojuego. Básicamente, esta medición se limita a comprobar si el jugador superó todas las misiones del juego, superó un test, etc. (Michael \& Chen, 2005). Por ejemplo, un juego en el que al final de cada misión se evalúa si ésta ha sido superada o no, o al final de todas las misiones se indica cuáles se han superado (Mayer et al., 2014).

- Teacher assessment: se basa en las observaciones producidas por el profesor o la persona responsable de realizar la evaluación (Michael \& Chen, 2005). Por ejemplo: un juego en el que una serie de expertos rellenan unas checklists en función del tiempo y luego le dan un peso para calcular la nota final (Khanal et al., 2014).

- Monitoring of states: este método permite llevar a cabo evaluaciones tanto sumativas como formativas asociando los diferentes estados del juego con la probabilidad de conseguir un objetivo de aprendizaje. Los estados pueden ser generales, como por ejemplo "nivel completado" o "respuesta correcta”, pero también podrían ser más específicos como "zona visitada” o "conversación con personaje no jugable iniciada” (Hainey et al, 2013; Chaudy et al., 2013). Al igual que con la evaluación InProcess, un buen ejemplo sería una aventura gráfica donde se monitoricen distintos tipos de estados para evaluar (Moreno-Ger et al., 2007).

- Quests: la evaluación es llevada a cabo a través de las búsquedas o misiones que componen el videojuego. El jugador suele ser evaluado tras la finalización de cada una de estas búsquedas o misiones, las cuales pueden ser de todo tipo (Hainey et al, 2013; Chaudy et al., 2013) Por ejemplo: un simulador en el que haya que completar una serie de objetivos (Mayer et al., 2014).

- Use of an assessment model or profile: este método de evaluación compara la actuación del jugador en el videojuego contra modelos o perfiles generales ya existentes: perfil de experto, perfil de conocimientos básicos, etc. También se incluyen en este método la implementación de modelos formales de pruebas, como por ejemplo tests formales de conductas o tests de detección de daltonismo (Hainey et al, 2013; Chaudy et al., 2013). Un ejemplo de juego que incluya esta evaluación sería un juego donde las acciones a realizar estén asociadas a un número y se compare el conjunto de números (por ejemplo, 145623) con el conjunto de números de un jugador experto (por ejemplo, 123456) (Loh \& Sheng, 2013).

- Non-invasive assessment: este enfoque busca evitar la presión a la que el estudiante puede verse sometido mientras juega a un videojuego sabiendo que está siendo evaluado, por lo que se evalúa al jugador sin darle ningún tipo de pista o feedback de su gameplay (al menos durante la partida). Además, en muchos de estos casos el propio juego puede adaptar la dificultad según la actuación del jugador (Hainey et al, 2013; Chaudy et al., 2013). Un videojuego que evalúe una serie de indicadores de forma interna sin dar feedback al usuario durante la partida se consideraría que incluye este método de evaluación (Raposo et al., 2013).

- Quizzes: este método de evaluación hace referencia a los videojuegos que tienen fases de pregunta/respuesta integrados durante todo o parte del juego (no solo al final). Los quizzes pueden ser de varios tipos: verdadero/falso, rellena los huecos, elección múltiple, etc. (Hainey et al, 2013; Chaudy et al., 2013). Por ejemplo, un juego con minijuegos de aprender idiomas donde haya que dar respuestas correctas o que haya que traducir palabras (Palomo-Duarte et al., 2016).

- Peer-assessment: este método incluye evaluación por compañeros, donde los jugadores se dan cierto status o feedback unos a otros (Hainey et al, 2013; Chaudy et al., 2013). Por ejemplo, un juego para aprender idiomas donde un jugador pueda evaluar a otro (Palomo-Duarte et al., 2016).

- Stealth assessment: en este método la evaluación es parte del propio gameplay del jugador y no interrumpe a éste en ningún momento de la experiencia de juego. Por ejemplo, se va recolectando "silenciosamente" todos los lugares visitados por el jugador para luego comprobar si los ha visitado todos (Shute, 2011). Un ejemplo de juego sería un juego donde se recolectasen una serie de indicadores de forma silenciosa durante el gameplay (Raposo et al., 2013).

\section{RESUlTADOS DEL ANÁLISIS SOBRE LOS MÉTODOS DE EVALUACIÓN}

De los métodos detectados, los 3 primeros (In-Process assessment, Completion assessment y Teacher assessment) pueden tomarse como métodos genéricos que no tienen en cuenta factores específicos de los videojuegos, ya que podrían aplicarse a cualquier otro tipo de actividad formativa. Según Bellotti (2013), el método In-Process assessment hace referencia a la evaluación formativa, mientras que el método Completion assessment hace referencia a la evaluación sumativa. De hecho, los propios autores comentan que estos tipos son considerados como "tipos principales de evaluación en serious games” (Michael \& Chen, 2005).

A diferencia de los anteriores, los métodos Monitoring of states, Quests, Use of an assessment model or profile, Noninvasive assessment, Quizzes y Peer-assessment sí que tienen en cuenta factores específicos de los videojuegos. En este caso con cómo se realiza la integración de la evaluación dentro de los propios juegos (Hainey et al, 2013; Chaudy et al., 2013), por lo que todos deben implementarse desde el propio juego y no con elementos externos.

Con el último método, Stealth assessment, se puede observar a través de su definición y ejemplo que es muy similar a Non-invasive assessment ya que ambos comparten el mismo objetivo: que el jugador no sepa que está siendo evaluado. De hecho, en la propia fuente el autor hace 
referencia al término "Embedded assessment” para referirse al mismo tipo de evaluación que presenta la "Stealth assessment”.

Se han proporcionado un ejemplo de serious game para cada método. Sin embargo, para algunos métodos se ha propuesto el mismo videojuego de ejemplo, ya que implementaba ambos tipos de evaluación. Estos métodos son los siguientes:

- In-Process assessment y Monitoring of states.

- Completion assessment y Quests.

- Non-invasive assessment y Stealth assessment.

- Quizzes y Peer-assessment.

Esto evidencia que los métodos no tienen por qué ser exclusivos entre sí y que en un mismo serious game pueden incluirse varios métodos de evaluación.

\section{CONCLUSIONES Y TRABAJOS FUTUROS}

Los serious games presentan verdadero potencial a la hora de diseñar procesos de aprendizaje ya que su naturaleza de juego conlleva particularidades como diversión o implicación que otros sistemas e-Learning no poseen (Shoukry et al., 2014). Por otro lado, a causa de la instauración de los sistemas de obtención de competencias, las estrategias de evaluación deben cambiar y tenerlas en cuenta. Sin embargo, la unión de estos dos temas, evaluación y serious games, no es un tema profundamente discutido en la literatura científica.

Se observa que los métodos detectados pueden ser generales o asociados a una particularidad de los serious games. Por tanto, se pueden clasificar las evaluaciones InProcess assessment, Completion assessment o Teacher assessment como métodos de evaluación principales o generales, mientras que Monitoring of states, Quests, Use of an assessment model or profile, Non-invasive assessment, Quizzes y Peer-assessment son métodos de integración de la evaluación dentro del videojuego. Por tanto, podrían existir en la literatura más métodos de evaluación en serious games asociados a otros factores, tanto generales como específicos.

No existe consenso sobre varios aspectos, como la terminología o métodos de evaluación establecidos, ya que se ha detectado cómo unos autores llaman Stealth assessment lo que otros entienden como Non-invasive assessment, o incluso Embedded assessment, compartiendo todos los mismos objetivos y características. Además, los métodos detectados no son de ninguna forma exclusivos, ya que un serious game puede ser evaluado por varios de estos métodos a la vez.

Vista la escasa de información y baja estandarización de métodos de evaluación en serious games, se antoja necesaria una revisión sistemática de la literatura para obtener una información más detallada y obtener resultados en profundidad, así como una clasificación de dichos resultados. Se detecta una falta de experiencias centradas en los métodos de evaluación en serious games que aumenta la necesidad de una revisión más profunda. Otra futura línea de investigación a destacar sería la realización de un caso de estudio para comparar la efectividad de los métodos de evaluación detectados.

\section{REFERENCIAS}

Abt, C. C. 1970. Serious games: The art and science of games that simulate life. USA: New Yorks Viking.

Balderas, A., Manuel Dodero, J., Palomo-Duarte, M., \& RuizRube, I. (2015). A Domain Specific Language for Online Learning Competence Assessments. International Journal of Engineering Education, 31(3), 851-862.

Bellotti, F., Kapralos, B., Lee, K., Moreno-Ger, P., \& Berta, R. (2013). Assessment in and of serious games: An overview. Advances in Human-Computer Interaction, 2013. doi:10.1155/2013/136864

Bente, G. \& Breuer, J. (2009). Making the implicit explicit: embedded measurement in serious games. In U. Ritterfield, M.J. Cody, \& P. Vorderer (Eds.), Serious Games: Mechanisms and Effects (pp. 322-343). New York, NY, USA: Routledge.

Berns, A., Isla-Montes, J. L., Palomo-Duarte, M., \& Dodero, J. M. (2016). Motivation, students' needs and learning outcomes: a hybrid game-based app for enhanced language learning. SpringerPlus, 5(1), 1305. doi:10.1186/s40064-016-2971-1

Buckingham, D. \& Scanlon, M. (2000). That is edutainment: media, pedagogy and the market place. Paper presented to the International Forum of Researchers on Young People and the Media, November 2000. Sydney, Australia.

Caballero-Hernández, J. A., Palomo-Duarte, M., Dodero, J. M., Rodríguez, G., \& Ibarra, M.S. (2014). Integrating external evidences of skill assessment in Virtual Learning Environments. In K. Jovanovic \& S. Jovanovic (Eds.), Proceedings of the Fifth International Conference on e-Learning (eLearning-2014) (pp. 6570). Belgrade, Serbia.

Chaudy, Y., Connolly, T., \& Hainey, T. (2013). Specification and Design of a Generalized Assessment Engine for GBL Applications. In 7th European Conference on Games Based Learning (pp. 105-114). Oporto: Instituto Superior de Engenharia do Porto (ISEP). Retrieved from http://www.researchgate.net/publication/257385262_Spe cification_and_Design_of_a_Generalized_Assessment_ Engine_for_GBL_Applications/file/60b7d̄5252ba4bd55e 1.pdf

Corti, K. (2006). Games-based Learning; a serious business application. Informe de PixelLearning, 34(6), 1-20.

Djaouti, D., Alvarez, J., \& Jessel, J. P. (2011). Classifying serious games: the G/P/S model. In P. Felicia (Ed.), Handbook of research on improving learning and motivation through educational games: Multidisciplinary approaches (pp. 118-136). Hershey, PA: IGI Global. doi:10.4018/978-1-60960-495-0.ch006

Enfield, J., Myers, D., Lara, M., \& Frick, T. W. (2012). Innovation diffusion: assessment of strategies within the diffusion simulation game. Simulation \& Gaming, 43(2), 188-214.

Graven, O. H., \& MacKinnon, L. (2005). A survey of current state-of-the art support for lifelong learning. In 6th International Conference on Information Technology 
Based Higher Education and Training 200 (pp. F2C/19F2C/25). doi:10.1109/ITHET.2005.1560277

Hainey, T., Connolly, T. M., Chaudy, Y., Boyle, E., Beeby, R., \& Soflano, M. (2013). Assessment Integration in Serious Games. In T. M. Connolly, T. Hainey, E. Boyle, G. Baxter, \& P. Moreno-Ger (Eds.), Psychology, Pedagogy, and Assessment in Serious Games (pp. 317341). Hershey, PA, USA: IGI Global. doi:10.4018/9781-4666-4773-2.ch015

Hodson, P., Connolly, M. \& Saunders, D. (2001) Can computer-based learning support adult learners? Journal of Further and Higher Education, 25(3), 325-335. doi:10.1080/03098770120077685

Ibarra, M. S., \& Rodríguez, G. (2011). Aprendizaje autónomo y trabajo en equipo: reflexiones desde la competencia percibida por los estudiantes universitarios. Revista Electrónica Interuniversitaria de Formación del Profesorado, 14, 73-85. Retrieved from http://www.redalyc.org/articulo.oa?id=217022117006

Khanal, P., Vankipuram, A., Ashby, A., Vankipuram, M., Gupta, A., Drumm-Gurnee, D., ... \& Smith, M. (2014). Collaborative virtual reality based advanced cardiac life support training simulator using virtual reality principles. Journal of biomedical informatics, 51, 49-59. doi:10.1016/j.jbi.2014.04.005

Loh, C. S., \& Sheng, Y. (2013). Performance metrics for serious games: Will the (real) expert please step forward?. In Proceedings of 2013 18th International Conference on Computer Games: AI, Animation, Mobile, Interactive Multimedia, Educational \& Serious Games (CGAMES) (pp. 202-206). doi:10.1109/CGames.2013.6632633

Mayer I., Zhou Q., Keijser X., Abspoel L. (2014) Gaming the Future of the Ocean: The Marine Spatial Planning Challenge 2050. In Ma M., Oliveira M.F., Baalsrud Hauge J. (Eds) Serious Games Development and Applications. SGDA 2014. Lecture Notes in Computer Science, 8778. Springer, Cham. doi:10.1007/978-3-31911623-5_13

Michael, D. \& Chen, S. (2005). Proof of Learning: Assessment in Serious Games. Gamasutra, 2005. Retrieved from http://www.gamasutra.com/view/feature/2433/proof_of_ learning_assessment_in_.php

Michael, D. \& Chen, S. (2006). Serious games: Games that educate, train, and inform. Boston, MA.: Thomson Course Technology.

Moreno-Ger, P., Burgos, D., Sierra, J. L., \& Manjón, B. F. (2007). A game-based adaptive unit of learning with IMS learning design and <e-Adventure>. In Duval E., Klamma R., Wolpers M. (Eds) Creating New Learning Experiences on a Global Scale. EC-TEL 2007. Lecture Notes in Computer Science, 4753 (pp. 247-261). Springer, Berlin, Heidelberg. doi:10.1007/978-3-54075195-3_18
Palés-Argullós, J., Nolla-Domenjó, M., Oriol-Bosch, a., \& Gual, a. (2010). Proceso de Bolonia (I): educación orientada a competencias. Educación Médica, 13(3), 127-135. doi:10.4321/S1575-18132010000300002

Palomo-Duarte, M., Berns, A., Cejas, A., Dodero, J. M., Caballero-Hernández, J. A., \& Ruiz-Rube, I. (2016). Assessing Foreign Language Learning Through Mobile Game-Based Learning Environments. International Journal of Human Capital and Information Technology Professionals (IJHCITP), 7(2), 53-67. doi:10.4018/IJHCITP.2016040104

Prensky, M. (2001). Digital game-based learning. USA: McGraw-Hill.

Raposo, F., Santos, G., \& Pereira, J. (2013). SimClinic-An Auxiliary Tool for Evaluation on Clinical Case Settings. In Ma M., Oliveira M.F., Petersen S., Baalsrud Hauge J. (Eds) Serious Games Development and Applications. SGDA 2013. Lecture Notes in Computer Science, 8101 (pp. 37-50). Springer Berlin Heidelberg. doi:10.1007/978-3-642-40790-1

Rodríguez, G., Ibarra, M. S., Dodero, J. M., Caballero, J. A., Cabeza, D., \& Ortega, C. (2012). Mejoras en la portabilidad de la integración de EvalCOMIX en Moodle 2. X. In M. Marco Such \& P. Pernías Peco (Eds.), Proceedings of the Ninth multidisciplinary symposium on the design and evaluation of digital content for education - SPDECE 2012 (pp. 81-91). Alicante, Spain.

Schmidt R., Emmerich K., Schmidt B. (2015) Applied Games - In Search of a New Definition. In: Chorianopoulos K., Divitini M., Baalsrud Hauge J., Jaccheri L., Malaka R. (Eds), Entertainment Computing - ICEC 2015. Lecture Notes in Computer Science, 9353 (pp. 100-111). Springer, Cham.

Shoukry, L., Göbel, S., \& Steinmetz, R. (2014). Learning analytics and serious games: trends and considerations. In Proceedings of the 2014 ACM International Workshop on Serious Games (pp. 21-26). New York, NY, USA: ACM. doi:10.1145/2656719.2656729

Shute, V. J. (2011). Stealth Assessment in Computer-Based Games To Support Learning. In S. Tobias \& J. D. Fletcher (Eds.), Computer Games and Instruction (pp. 503-524). Cambridge: MIT Press.

Traverso-Ribón, I., Balderas-Alberico, A., Dodero, J. M., Ruiz-Rube, I., Palomo-Duarte, M. (2016). Open data framework for sustainable assessment of project-based learning experiences. Program, 50(4), 380-398. doi: 10.1108/PROG-12-2015-0078

Van Eck, R. (2006). Digital game-based learning: It's not just the digital natives who are restless. EDUCAUSE review, 41(2), 16-30.

Zyda, M. From visual simulation to virtual reality to games. Computer (Long. Beach. Calif)., 38(9), 25-32. doi:10.1109/MC.2005.297 